\title{
LAS EXPORTACIONES ANDALUZAS
}

Gumersindo RUIZ BRAVO de MANSILLA *

Antonio RUIZ MOLINA *

\section{INTRODUCCION: CONSIDERACIONES METODOLOGICAS}

El análisis que aquí se realiza pretende sólo presentar los datos de exportaciones de una forma simple, destacando aquellos aspectos de las mismas que mejor puedan ilustrar la realidad actual de la exportación andaluza.

Analizar las peculiaridades de las mismas, las características de los sectores, sus posibilidades futuras, requiriría ampliar el estudio a otras variables productivas, y de demanda, que superan el ámbito de este trabajo, pues es claro que las exportaciones constituyen un indicador de actividad productiva competitiva, pero resultan por sí solas un dato insuficiente para conocer la situación y perspectivas de un sector.

Para nuestro trabajo hemos contado con experiencias realizadas anteriormente en las que destacan las dificultades metodológicas de aproximación a las exportaciones regionales**. Esto permite una primera aproximación a las ventajas e inconvenientes de las distintas fuentes estadísticas existentes.

La elección de los datos suministrados por la Dirección General de Aduanas se apoya, pues, en estos criterios, aunque los datos que nos han facilitado han debido ser depurados.

En primer lugar, hemos de resaltar que los datos base presentan la limitación de que para aquellas empresas con sede social distinta de cualquiera de las provincias integrantes de la región andaluza, los datos de sus exportaciones se computan en las provincias donde tal sede social se ubica, y por el

* Profesores de la Facultad de Ciencias Económicas de la Universídad de Málaga, Aunque estas páginas han sido redactadas por quienes las fírman, este trabajo es parte de uno más amplio sobre las exportaciones andaluzas, realizado en colaboración con la Jefatura de Estudios de la Consejerfa de Economía, Planificación. Industria y Energfa; la corresponsabilidad del estudio pertenece a Doña Rosario Conde, así como a los Profesores Joaquín Auroles, Antonio Narváez, José Tomás Soria y Francisco Trigueros, quien se ocupó del programa de ordenador.

* Como el que realizan Aurelio Martínez Esteve, Fernández Guerrero y Sanchis Marco en Dinámica exportadora del Pafs Valenciano. 
contrario, para cualquier empresa con sede social en Andalucía que mantenga factorías o sucursales fuera de la región, se consideran las exportaciones realizadas a través de tales sucursales como propias de la región.

Una vez tenidos en cuenta ambos problemas, se llegó a la conclusión de que el segundo apenas tiene actualmente incidencia, mientras el primero sí representa un volumen importante en el total de exportaciones de la empresa, que no quedaba recogido en los datos oficiales y que, por tanto, minusvalora las exportaciones andaluzas. En base a este criterio se procedió a la obtención de datos acerca de cuáles eran las empresas exportadoras con sede social fuera de Andalucía y que al mismo tiempo mantuviesen actividad productiva en nuestra región; para, en un segundo paso, llegar a determinar con cierta exactitud cual es la parte de la exportación de esas empresas, que se produce en Andalucía.

Para el conocimiento de las empresas que desarrollan actividad productiva para la exportación en la región, y que no venían incluidas en los listados suministrados por la Dirección General de Aduanas, se utilizaron diversas fuentes, entre las que podemos destacar:

- La relación de empresas utilizada por el equipo que ha confeccionado las TIO-80 de Andalucía y que recoge todas aquellas existentes en Andalucía que mantienen un número de trabajadores superior a $20 \mathrm{y}$ que, al mismo tiempo, realizan desde Andalucía exportaciones.

- Datos recogidos de publicaciones de Cámaras de Comercio e Industria de la región, en que se relacionan todas las empresas con actividad exportadora.

- Datos recogidos en las publicaciones Ministerio de Economía y Comercio del I.N.F.E., en que se relacionan las empresas exportadoras cuyo volumen de exportación es superior a los 10.000 .000 pts. y la relación de empresas más importantes que de cada producto exporta el 75 por ciento del total nacional exportado.

Una vez contrastados los datos originarios con las distintas fuentes, y conocidas cuáles eran las empresas que desarrollaron actividad exportadora para los años 1980, 1981 y 1982 y no estaban incluidas en los listados primarios, se observó lo siguiente. De un lado, cómo un conjunto de empresas que tenían su sede central en Andalucía y debían estar incluidas en los listados, no se encontraban; esto se producía fundamentalmente para el año 1980 y en menor medida para 1981, siendo este problema minúsculo en el año 1982 (todo ello consecuencia de que los datos suministrados por la Dirección General de Aduanas empezaron a recopilarse a partir de 1980 con la consiguiente mejora año tras año). De otro lado, se llegó al conocimiento de cuáles eran las empresas que realizaban actividad exportadora y ésta se recogía en otras provincias. 
El primer caso expuesto se solventó en base a los datos recogidos de las encuestas, antes mencionadas, realizadas para la confección de las TIO-80.

Para el segundo problema, y realmente es el más importante por el volumen de exportaciones realizado por tales empresas, nos sirvieron de base en primer lugar las encuestas anteriormente citadas y, en segundo lugar, un cuestionario que se envió a cada una de las empresas restantes en que se indicaba cual era el porcentaje de sus exportaciones totales que había tenido su origen en actividad productiva realizada en Andalucía.

Una vez se obtuvieron los datos referentes al volumen de exportaciones de las empresas con sede social fuera de Andalucía, surgió el problema de su distribución geográfíca, para lo cual adoptamos el supuesto de considerar que ésta era la misma existente en el total nacional de dichas empresas.

El trabajo cubre el período 1980-82.

\section{PRODUCTOS Y AREAS DE DESTINO}

Como se ve en el cuadro I, los diez primeros capítulos de exportación suponen el 61,17 por ciento de las exportaciones, para el trienio 1980-82. De ellos, los cinco primeros representan el 41,74 por ciento de ese total. Puede considerarse, pues, que hay una cierta diversificación de exportaciones, aunque aparezcan mayoritariamente concentradas en los capítulos que figuran en el cuadro I.

\section{CUADRO I}

\begin{tabular}{|c|c|c|c|c|}
\hline $\begin{array}{l}S \\
(1)\end{array}$ & $\begin{array}{l}N \\
(2)\end{array}$ & $\begin{array}{l}\text { Diez principales capítulos. } \\
\text { Trienio } 1980-1982\end{array}$ & $\begin{array}{c}\% / 0 \\
\text { Total } \\
\text { exportaciones }\end{array}$ & $\begin{array}{l}\text { Tendencia } \\
\text { (3) }\end{array}$ \\
\hline IV & 22 & Bebidas, líquldos alcohólicos y vinagre. . . . . & 10,37 & LD \\
\hline $\mathrm{v}$ & 27 & Combustibles y aceites minerales . . . . . . & 9,70 & FC \\
\hline XVII & 89 & Material de navegación marítima y fluvial .... & 9,48 & LC \\
\hline IV & 20 & Preparados de legumbres, hortallzas, frutas, etc. . & 6,81 & c \\
\hline $\mathrm{XV}$ & 73 & Fundición de hierro y acero $\ldots \ldots \ldots \ldots$ & 5,38 & LC \\
\hline XVII & 87 & Vehŕculos automóviles, tractores, etc. . . . . & 4,60 & $\mathbf{E}$ \\
\hline $\mathrm{xV}$ & 74 & Cobre. . . . . . . . . . . . . . & 4,07 & LD \\
\hline III & 15 & Grasas y aceites $\ldots \ldots \ldots \ldots \ldots \ldots$ & 3,83 & FD \\
\hline $\mathrm{v}$ & 26 & Minerales metalúrgicos $\ldots \ldots \ldots \ldots \ldots$ & 3,75 & LC \\
\hline $\mathbf{x}$ & 47 & Materfas utilizadas en la fabricación de papel . . & 3,18 & LD \\
\hline
\end{tabular}

(1) Sección a la que corresponde.

(2) Nomenclatura de Bruselas.

(3) Significado en el texto. 
La tendencia observada en el trienio 1980-82 resulta obviamente insuficiente para extraer alguna conclusión acerca de la evolución probable de las exportaciones. Por este motivo hemos realizado una aproximación a la tendencia observada en dichos años, que se muestran fuertemente creciente (FC) en el grupo 27, de "Combustibles y aceites minerales"; creciente (C), en el 20, "Preparados de legumbres, hortalizas y frutas"; ligeramente crecientes (LC), 73, "Fundición de hierro y acero", 26 "Minerales metalúrgicos" y 89 "Material de navegación marítima y fluvial"; ligeramente decrecientes (LD), 22, "Bebidas, líquidos alcohólicos y vinagre", 74 "Cobre", 47 "Materias utilizadas en la fabricación de papel"; y fuertemente decrecientes (FD), 15, "Grasas y aceites".

Estas tendencias nos indican que salvo dos casos, de fuerte crecimiento y fuerte decrecimiento, las exportaciones de los productos señalados mantienen una cierta constancia (con ligeras variaciones al alza o la baja) en el período estudiado, en su participación en el total de exportaciones.

Tal como aparece en el cuadro II, si agrupamos por secciones los 99 grupos de productos, vemos que las tres primeras secciones en cuanto a porcentaje de exportación sobre el total, suponen el 50 por ciento $(50,50$ por cien) de las exportaciones. Destaca así la importancia de los "Productos de industrias alimenticias", "Material de transporte" y "Productos minerales". Hemos consignado otras nueve secciones en orden decreciente de importancia, agrupando en "Resto" aquellos que no legan a representar el 1 por ciento.

\section{CUADRO II}

PRINCIPALES SECCIONES DE EXPORTACIONES 1980-1982

EN \% DEL TOTAL

Productos industrias alimenticias. . . . . . . . .

$(17,64)$

XVII

Material de transporte.

$\mathrm{V}$

Productos minerales

VI

Productos de industrias químicas . ..........

$(10,44)$

XV

Metales y sus manufacturas.

II

Productos vegetales . . . . . . . . . . . . . .

Materias fabricación de papel $\ldots \ldots \ldots \ldots \ldots \ldots$

Grasas y aceites . . . . . . . . . . . . . .

XVI

Perlas, piedras y metales preciosos y semipreciosos, chapado y bisuterfa..................

Animales y productos animales...$\ldots \ldots \ldots \ldots$

Materiales y manufacturas textiles...........

Restantes secciones ................. 
No se observa una fuerte concentración por secciones de exportaciones, aunque sí es obvia la especialización en productos extractivos (agricultura y minería) y la transformación primaria de los mismos. Junto a ellos, aparecen algunos enclaves como el "Material de transporte", los "Productos de industrias químicas" y en mucha menor medida, "Máquinas y aparatos; material eléctrico", que no logran hacer posible calificar la exportación de manufacturera. Se advierte la ausencia de exportaciones significativas en secciones tan importantes como la VII, "Materias plásticas artificiales", o la XVIII, "Instrumentos y aparatos de precisión", así como de los capítulos de la sección XX, "Mercancías y productos varios", que incluye mobiliario, juguetes, artículos de deportes; en este sentido, obsérvese también la escasa importancia de la XI, "Materiales y manufacturas textiles", un sector que podría corresponder a la estructura exportadora de una economía en desarrollo.

La distribución porcentual de las principales secciones aparece en el cuadro III. El él puede comprobarse que los capítulos de la sección IV, "Productos de industrias alimenticias" (que incluye el vino), se venden principalmente a Europa (55 por ciento) y a América del Norte (27,06 por ciento).

El grupo XVII: "Material de transporte", se destina a Europa $(21,18$ por ciento). América del Sur y Centro $(24,20)$, Asia $(18,41)$ y Resto del Mundo $(21,62)$.

Del grupo V: "Productos minerales", son nuestros principales compradores Europa $(42,32$ por ciento), Resto del Mundo $(16,02)$ y Norte de Africa $(13,44)$.

El VI: "Productos de industrias químicas", lo adquieren Europa (50,32 por ciento), Asia $(10,83)$ y América del Sur y Centro $(10,63)$.

El grupo XV: "Metales y manufacturas de metales", va a Europa $(59,14$ por ciento), Asia $(13,39)$, Resto del Mundo $(9,80)$ y Norte de Africa $(8,92)$.

El II: "Productos vegetales", va a Europa (63,74 por ciento) y Norte de Africa $(22,36)$.

El X: "Materias para la fabricación de papel", a Europa (70,37 por ciento), Resto del Mundo $(11,95)$ y América del Sur y Centro $(9,11)$.

El III: "Grasas y aceites", al Norte de Africa (30,04 por ciento), Resto del Mundo $(13,62)$, Europa $(19,79)$ y América del Norte $(16,03)$.

El XVI: "Máquinas y aparatos, material eléctrico", a Europa $(53,02$ por ciento) y América del Sur y Centro $(25,28)$.

El I: "Animales y productos animales", a Europa (55,53 por ciento), Asia $(25,47)$ y Resto de Africa $(10,20)$.

El XI: "Materiales y manufacturas textiles", a Europa $(74,10$ por ciento).

Las secciones que no se dirigen mayoritariamente a Europa, la XVII: "Material de transporte" y la III: "Grasas y aceites", son las más diversificadas en cuanto que se dirigen en porcentajes apreciables a todas las áreas geográficas consideradas. 
CUADRO III

DISTRIBUCION PORCENTUAL DE LAS PRINCIPALES SECCIONES POR AREAS DE DESTINO

\begin{tabular}{|c|c|c|c|c|c|c|c|c|c|c|}
\hline & Europa & $\begin{array}{l}\text { América Sur } \\
\text { y Centro }\end{array}$ & $\begin{array}{l}\text { América } \\
\text { Norte }\end{array}$ & $\begin{array}{l}\text { Norte } \\
\text { Africa }\end{array}$ & $\begin{array}{l}\text { Resto } \\
\text { Africa }\end{array}$ & $\begin{array}{c}\text { Países } \\
\text { Este }\end{array}$ & Asia & $\begin{array}{l}\text { Resto } \\
\text { Mundo }\end{array}$ & Otros & $\begin{array}{l}\text { Total } \\
(\% / 0)\end{array}$ \\
\hline IV $\ldots \ldots \ldots \ldots$ & 55,00 & 5,11 & 27,06 & 0,34 & 2,33 & 0,98 & 2,68 & 6,49 & 0,02 & 100 \\
\hline 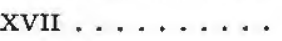 & 21,18 & 24,20 & 2,77 & 7,53 & 1,80 & 3,22 & 18,41 & 21,62 & 0,00 & 100 \\
\hline$v \ldots \ldots$ & 42,32 & 1,83 & 0,91 & 13,44 & 5,93 & 2,06 & 7,60 & 16,02 & 9,88 & 100 \\
\hline 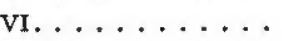 & 50,32 & 10,63 & 7,00 & 6,32 & 1,59 & 3,97 & 10,83 & 8,92 & 0,44 & 100 \\
\hline $\mathrm{xV} \ldots \ldots \ldots$ & 59,14 & 3,77 & 1,69 & 8,92 & 2,65 & 0,02 & 13,99 & 9,80 & 0,01 & 100 \\
\hline $\mathrm{II} \ldots \ldots \ldots$ & 63,74 & 1,42 & 1,76 & 22,36 & 1,16 & 1,12 & 5,21 & 3,22 & 0,02 & 100 \\
\hline$x \ldots \ldots$ & 70,37 & 9,11 & 0,98 & 4,08 & 0,06 & 0,35 & 1,12 & 11,25 & - & 100 \\
\hline III. . . . . . . . . & 19,79 & 4,22 & 16,03 & 30,04 & 3,54 & 5,27 & 7,45 & 13,62 & - & 100 \\
\hline XVI. ......... & 53,02 & 25,28 & 2,59 & 6,60 & 3,23 & 0,01 & 4,70 & 4,49 & 0,04 & 100 \\
\hline 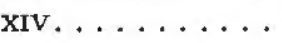 & 92,75 & 0,42 & 4,51 & 0,00 & 0,23 & 0,34 & 0,02 & 1,71 & 0,00 & 100 \\
\hline 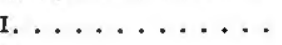 & 55,53 & 2,08 & 6,35 & 0,00 & 10,20 & 0,00 & 25,47 & 0,18 & 0,18 & 100 \\
\hline $\mathrm{xI} \ldots \ldots \ldots$ & 74,10 & 4,34 & 2,79 & 3,12 & 5,70 & 0,71 & 4,03 & 5,17 & 0,01 & 100 \\
\hline Todas Ias secciones. . & 48,29 & 9,12 & 7,76 & 8,41 & 2,82 & 1,79 & 9,23 & 10,95 & 1,53 & - \\
\hline
\end{tabular}


Se comprueba claramente la decisiva importancia de Europa, que, en conjunto recibe el 50 por ciento de las exportaciones andaluzas. De todas las secciones consideradas básicas en las exportaciones andaluzas, Europa recibe el 50 por ciento o más, excepto la V: "Productos minerales" $(42,32$ por ciento); XVII: "Material de transporte" (26,26 por ciento) y III: "Grasas y aceites" (19,79 por ciento), aunque en la V y prácticamente en la XVII, continúa siendo el principal importador.

La situación se caracteriza, pues, por la existencia de un área: Europa, a la que se dirige el volumen principal de exportaciones de un grupo heterogéneo de capítulos que poseen las dos características señaladas: productos extractivos escasamente elaborados y transformados, y enclaves industriales que se utilizan para la exportación.

Junto a estas circunstancias se muestra un cierto decrecimiento en el período de tres años considerado (ver cuadro IV), aunque se confirma que la existencia de un mercado con capacidad de demanda sólidamente establecida es la opción exportadora más clara.

\section{CUADRO IV}

DISTRIBUCION PORCENTUAL DE LAS EXPORTACIONES POR AREAS DE DESTINO

\begin{tabular}{|c|c|c|c|c|c|}
\hline & $\begin{array}{c}\text { Trienio } \\
80 / 82\end{array}$ & 1980 & 1981 & 1982 & $\begin{array}{c}\text { Tendencia } \\
\left({ }^{*}\right)\end{array}$ \\
\hline Europa $\ldots \ldots \ldots \ldots \ldots$ & 49,99 & 51,26 & 49,42 & 45,30 & $\mathrm{D}$ \\
\hline América Sur y Centroamérica ... & 9,12 & 9,83 & 8,95 & 8,97 & E \\
\hline América Norte. . . . . . . . & 7,76 & 6,51 & 8,35 & 8,18 & $\mathrm{C}$ \\
\hline Norte Africa . . . . . . . . & 8,41 & 10,97 & 7,22 & 7,54 & 1 \\
\hline Resto Africa. . . . . . . & 2,82 & 2,58 & 3,96 & 2,07 & 1 \\
\hline Países Este . . . . . . . & 1,79 & 2,85 & 1,72 & 1,09 & $\mathrm{D}$ \\
\hline Asia. . . . . . . . & 9,23 & 7,72 & 8,52 & 10,87 & c \\
\hline Resto Mundo $\ldots \ldots \ldots$ & 10,95 & 6,63 & 8,53 & 15,96 & $\mathrm{C}$ \\
\hline Otros $\ldots \ldots \ldots \ldots \ldots \ldots$ & 1,53 & 1,64 & 3,33 & 0,01 & - \\
\hline Total en $\% \ldots \ldots \ldots$ & 100,00 & 100,00 & 100,00 & 100,00 & - . \\
\hline
\end{tabular}

(*) E, Estable; D, Decreciente; C, Creciente; I, Inestable.

La significación de las áreas "Resto de Africa" y Países del Este" es muy escasa; la primera se mueve con una cierta irregularidad a bajo nivel y la segunda con una tendencia claramente decreciente (ver cuadro IV). 
Resulta peculiar que las restantes áreas participen aproximadamente en el mismo porcentaje ( 8 a 9 por ciento) del total de exportaciones, pero mientras "América del Sur y Centroamérica" refleja una tendencia estable (pese a la situación económica de estos países) y el "Norte de Africa", una acusada inestabilidad, "América del Norte", "Asia" y "Resto del Mundo" crecen en su participación, siendo especialmente significativo al aumento de este último.

¿Qué tipo de productos se exportan a estas áreas?. Por las características de nuestras exportaciones (primeras materias con escasa elaboración y enclaves industriales para la exportación), no hay una forma específica de exportaciones, que pueda hacerse corresponder a un área concreta. El mercado de "América del Norte" recibe productos de la sección IV: "Preparados de legumbres, hortalizas, frutas..." y "Bebidas, vinos", respectivamente. Y también la sección III: "Grasas y aceites". En menor medida, el capítulo 32: "Colorantes y tintes" (de la sección VI) y el 88: "Navegación aérea" (dentro de la sección XVII: "Material de transporte").

Las cuatro áreas que pueden considerarse de países en desarrollo: "América del Sur y Centro", "Norte de Africa", "Asia" y "Resto del Mundo" adquieren porcentajes significativos de las secciones XVII: "Material de transporte" (en sus distintas clases de carretera, aéreo y marítimo) y de la VI: "Productos minerales" y la XV: "Metales y manufacturas de metales", se dirigen también de forma importante a esas áreas (exceptuando ahora "América del Sur y Centro").

"América del Sur y Centro" adquiere un elevado porcentaje $(24,20$ por ciento) de la sección XVII: "Material de transporte" (navegación aérea y, sobre todo, marítima), de la VI (10,63 por ciento): "Industrias químicas", X (9,11 por ciento): "Materias de fabricación de papel" y de la XVI $(25,28$ por ciento): "Máquinas y aparatos; material eléctrico".

El "Norte de Africa" participa en porcentajes notables de la sección V: "Productos minerales" (13,44 por ciento): XVII: "Material de transporte" (8,06 por ciento); XV: "Metales y sus manufacturas" (8,92); II: "Productos vegetales" (22,36 por ciento) (por una importante cantidad del capítulo n.o 11: "Productos de la molinería") y III: "Grasas y aceites" $(30,04)$.

"Asia" supone el 17,07 por ciento de la sección XVIII: "Material de transporte" (tanto en terrestre, como en aéreo y marítimo); el 10,83 de la VI: "Productos de industrias químicas"; el 13,99 por ciento del XV: "Metales y sus manufacturas"; y el 25,47 por ciento de la I: "Animales y productos animales" (producto n. 0 3: "Pescados, crustáceos y moluscos").

En el "Resto del Mundo" se encuadran porcentajes significativos, que indican cierta desconcentración de esos productos, si bien no hay que olvidar los porcentajes tan elevados que van a los países europeos. Destaca el grupo V: "Productos minerales" (16,02 por ciento); el XVII: "Material de transporte" (13,86 por ciento); el VI: "Productos de industrias químicas" (8,92 por ciento); el XV: "Metales y sus manufacturas" ( 9,80 por ciento); el X: 
"Materias de la fabricación del papel" (11,95 por ciento) y el III: "Grasas y aceites" (13,62 por ciento).

Hay que distinguir si un área importa un elevado porcentaje del total de exportaciones de un producto o capítulo, o bien si se trata de una cantidad monetariamente apreciable, pues ambas cosas no tienen por qué coincidir, ya que un área puede importar un elevado porcentaje, pero de un producto o capítulo que en su total no resulte importante.

En el caso en que un área domine porcentualmente el mercado de un producto, se nos indica una posición de dependencia respecto al producto de que se trate. En caso de que el porcentaje sea bajo, pero la cantidad apreciable, estamos ante un mercado que por el volumen que representa, conviene cuidar.

En el cuadro $V$ se recoge esta distinción. Así, por el volumen de las cuatro primeras secciones destaca "Europa", cuyas partidas suponen cantidades que sobrepasan ampliamente las de cualquier área.

"América del Sur y Centro" destaca en "Material de transporte" (XVII), siguiendo a considerable distancia las secciones VI, IV y XVI.

En "América del Norte" es significativa por su volumen las importaciones de "Productos de industrias alimenticias" (IV), y en mucho menor grado las secciones VI, III y XVII.

El "Norte de Africa" muestra una mayor homogeneidad, por la no existencia de secciones que supongan volúmenes de importación considerables y la relativa aproximación entre ellos. Algo similar ocurre con "Asia", donde la primera partida: "Material de transporte" (XVII) es el doble que la XV y tres veces más que la $\mathrm{V}$ y VI. Y asimismo tiene lugar en las exportaciones al "Resto del Mundo", con dos partidas significativas: "Material de transporte" (XVII) y "Productos minerales" (V).

En suma, salvo alguna sección ocasional, que se corresponde con el primer capítulo de exportación a cada una de las áreas: "Material de transporte" (XVII) ("América del Sur y Centro" y "Asia"), "Industrias alimenticias" (IV) ("América del Norte"), "Productos minerales" (V) ("Norte de Africa" y "Resto del Mundo"), los volúmenes más importantes se dirigen a Europa, donde los "Productos de industrias alimenticias" (IV), "Productos minerales" (V), "Metales y sus manufacturas" (XV) y "Productos de industrias químicas" (VI), ocupan cuantitativamente los primeros lugares.

Como decíamos al principio, en estas páginas nos hemos limitado a una descripción de la situación. Un análisis que pretendiera extraer consecuencias sobre los sectores productivos obviamente no podría basarse únicamente en la característica exportadora de los mismos. El supuesto de que un sector es productivo y competitivo porque exporta (esto es, es un sector abierto a una competencia internacional) no es suficiente para decidir sobre una política de apoyo a sectores establecidos, o para dilucidar qué actividades productivas conviene estimular. Sería preciso realizar un estudio en profundidad de cada 


\section{CUADRO V \\ LAS PRINCIPALES SECCIONES (CUANTIT ATIVAMENTE) DE CADA AREA (Trienio 1980-1982. Millones de pts.)}

\section{Europa}

IV. "Productos industrias alimenticias". . . . . . . . . . . 53.940

v. "Productos minerales" ....................... 35.008

XV. "Metales y sus manufacturas". ................... 33.848

vi. "Productos industrias químicas" . . . . . . . . . . . . 29.194

\section{Anérica del Norte}

IV. "Productos industrias alimenticias". . . . . . . . . . . . 26.539

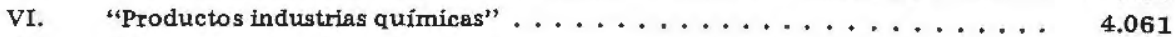

III. "Grasas y aceites". . . . . . . . . . . . . . . . . 3.412

XVII. "Materias de transporte". .................... 2.704

\section{Asia}

XVII. "Material de transporte".................... 17.936

XV. "Metales y sus manufacturas" .................. 8.007

V. "Productos minerales" . . . . . . . . . . . . . . . 6.285

VI. "Productos industrias químicas" ................ 6.283

\section{América Sur y Centro}

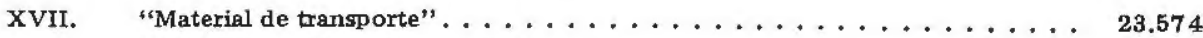

VI. "Productos industrias químicas" ................ 6.167

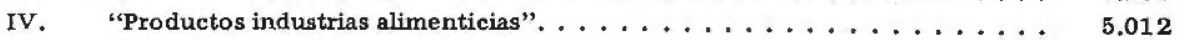

XVI. "Máquinas y aparatos". ..................... 4.748

Norte Africa

v. "Productos minerales" ..................... 11.118

II. "Productos vegetales" . . . . . . . . . . . . . . . . . 9.194

III. "Grasas y aceites". ....................... 6.394

XVII. "Material de transporte" .................... 6.334

Resto Mundo

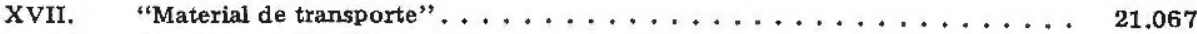

V. "Productos minerales" . . . . . . . . . . . . . . . . 13.253

IV. "Productos industrias alimenticias". . . . . . . . . . . 6.362

XV. "Metales y sus manufacturas". ................. 5.607 
sector concreto atendiendo a su funcionamiento empresarial y microeconómico y, de manera específica, la evolución, en el contexto internacional, de ese sector.

De todas formas, los datos que aquí figuran sobre exportaciones andaluzas, ponen de manifiesto algunos rasgos que sirven para informar las líneas de una política. Así, se hace patente el carácter de exportador de primeras materias, con escasa elaboración, y el enclave de algunas actividades industriales escasamente articuladas en la estructura productiva interna, dirigidas a la exportación. A un área de fuerte demanda como es Europa, se dirige el 50 por ciento de las exportaciones; el otro 50 por ciento se reparte equilibradamente por todo el mundo, indicando una no excesiva dependencia.

Este último dato, junto con la tampoco excesiva concentración de los productos (aunque no hay que olvidar que los diez primeros suponen el 60 por ciento de las exportaciones), permiten considerar con optimismo las posibilidades de potenciar las actividades existentes y lograr una industria competitiva e integrada en los mercados internacionales.

\section{DISTRIBUCION PROVINCIAL DE LAS EXPORTACIONES}

El cuadro VI muestra una fuerte concentración de las exportaciones en las provincias de Cádiz, Sevilla y Huelva, que suponen el 80 por ciento, con una muy reducida participación de las restantes provincias en el total. La participación de Almería en los tres años es ligeramente creciente, creciente la de Cádiz y fuerte la de Sevilla. Decrecen las otras cinco provincias, aunque Huelva mantiene un elevado porcentaje de participación.

\section{CUADRO VI}

EXPORTACIONES POR PROVINCIAS

$(\%)$

\begin{tabular}{|c|c|c|c|c|}
\hline & 1980 & 1981 & 1982 & Tres años \\
\hline Almería . . . . . . . . & 3,35 & 2,97 & 3,74 & 3,38 \\
\hline 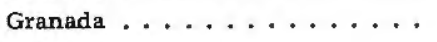 & 2,33 & 1,84 & 2,16 & 2,11 \\
\hline Jaén. . . . . . . . . . & 7,81 & 5,56 & 4,30 & 5,69 \\
\hline Málaga . . . . . . . . . . & 5,76 & 4,51 & 3,27 & 4,37 \\
\hline Andalucía Oriental . . . . . & 19,25 & 14,89 & 13,47 & 15,55 \\
\hline Cádiz . . . . . . . . . . & 30,68 & 32,90 & 35,07 & 33,14 \\
\hline 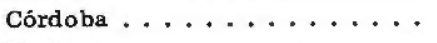 & 6,37 & 3,91 & 3,44 & 4,42 \\
\hline Huelva . . . . . . . . . & 24,29 & 23,79 & 18,91 & 21,99 \\
\hline 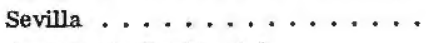 & 19,41 & 24,51 & 29,11 & 24,90 \\
\hline Andalucía Occidental. . . . . & 80,11 & 85,11 & 86,53 & 84,45 \\
\hline
\end{tabular}




\section{CUADRO VII}

DISTRIBUCION DE LAS PRINCIPALES SECCIONES POR PROVINCIAS. TRIENIO 1980-82

(Millones de pesetas y porcentajes)

\begin{tabular}{|c|c|c|c|c|c|c|c|c|c|c|}
\hline & & & Almería & Granada & Jaên & Málaga & Cádiz & Córdoba & Huelva & Sevilla \\
\hline IV. & $(100 \%$ & )$\ldots \ldots$ & $\begin{array}{c}1.122,0 \\
(1,14)\end{array}$ & $\begin{array}{l}100,0 \\
(0,10)\end{array}$ & $\begin{array}{c}450,7 \\
(0,46)\end{array}$ & $\begin{array}{r}2,264,5 \\
(2,31)\end{array}$ & $\begin{array}{c}54.487,0 \\
(55,56)\end{array}$ & $\begin{array}{c}3.024,1 \\
(3,08)\end{array}$ & $\begin{array}{c}267,5 \\
(0,27)\end{array}$ & $\begin{array}{c}36.349,1 \\
(37,06)\end{array}$ \\
\hline XVII. & $"$ & $\ldots \ldots$ & $\begin{array}{l}171,0 \\
(0,18)\end{array}$ & $\begin{array}{r}75,1 \\
(0,08)\end{array}$ & $\begin{array}{c}12.192,4 \\
(42,51)\end{array}$ & $\begin{array}{c}108,2 \\
(0,11)\end{array}$ & $\begin{array}{c}41.643,3 \\
(42,64)\end{array}$ & $\begin{array}{r}23,9 \\
(0,02)\end{array}$ & $\begin{array}{c}640,1 \\
(0,66)\end{array}$ & $\begin{array}{c}42,683,0 \\
(43,81)\end{array}$ \\
\hline V. & $"$ & $\ldots \ldots$ & $\begin{array}{c}297,1 \\
(0,36)\end{array}$ & $\begin{array}{c}3.219,1 \\
(3,39)\end{array}$ & $\begin{array}{r}42,1 \\
(0,05)\end{array}$ & $\begin{array}{c}3.328,7 \\
(4,02)\end{array}$ & $\begin{array}{c}41.852,4 \\
(50,59)\end{array}$ & $\begin{array}{c}1.297,8 \\
(1,57)\end{array}$ & $\begin{array}{c}23,662,7 \\
(28,60)\end{array}$ & $\begin{array}{l}9.022,6 \\
(10,91)\end{array}$ \\
\hline VI. & $"$ & $\ldots \ldots$ & $\begin{array}{c}1.971,0 \\
(3,40)\end{array}$ & $\begin{array}{c}1.166,9 \\
(2,01)\end{array}$ & $\begin{array}{c}12,1 \\
(0,02)\end{array}$ & $\begin{array}{c}816,8 \\
(1,41)\end{array}$ & $\begin{array}{c}15.792,4 \\
(27,22)\end{array}$ & $\begin{array}{c}147,6 \\
(0,25)\end{array}$ & $\begin{array}{c}35.017,8 \\
(60,36)\end{array}$ & $\begin{array}{c}3.093,9 \\
(5,33)\end{array}$ \\
\hline $\mathrm{XV}$ & $"$ & $\cdots \cdots$ & $\begin{array}{r}0,0 \\
(0,00)\end{array}$ & $\begin{array}{r}47,6 \\
(0,08)\end{array}$ & $\begin{array}{c}343,2 \\
(0,60)\end{array}$ & $\begin{array}{c}1.968,4 \\
(3,44)\end{array}$ & $\begin{array}{c}10.761,3 \\
(18,80)\end{array}$ & $\begin{array}{c}1.193,9 \\
(2,09)\end{array}$ & $\begin{array}{c}22.124,1 \\
(38,66)\end{array}$ & $\begin{array}{c}20.795,3 \\
(36,33)\end{array}$ \\
\hline II. & $"$ & $\ldots$ & $\begin{array}{c}14.600,6 \\
(35,51)\end{array}$ & $\begin{array}{l}4.218,5 \\
(10,26)\end{array}$ & $\begin{array}{l}8.464,2 \\
(20,59)\end{array}$ & $\begin{array}{c}2,971,4 \\
(7,23)\end{array}$ & $\begin{array}{c}836,3 \\
(2,03)\end{array}$ & $\begin{array}{c}857,4 \\
(2,09)\end{array}$ & $\begin{array}{c}267,8 \\
(0,65)\end{array}$ & $\begin{array}{l}8.900,1 \\
(21,65)\end{array}$ \\
\hline $\mathrm{x}$. & $"$ & $\cdots \cdots$ & $\begin{array}{c}13,8 \\
(0,06)\end{array}$ & $\begin{array}{c}2.123,1 \\
(8,90)\end{array}$ & $\begin{array}{c}67,5 \\
(0,24)\end{array}$ & $\begin{array}{r}50,2 \\
(0,21)\end{array}$ & $\begin{array}{l}2.648,5 \\
(11,11)\end{array}$ & $\begin{array}{c}1.251,7 \\
(5,25)\end{array}$ & $\begin{array}{c}17.380,5 \\
(72,89)\end{array}$ & $\begin{array}{r}319,8 \\
(1,34)\end{array}$ \\
\hline III. & $"$ & $\ldots \ldots$ & $\begin{array}{r}0,0 \\
(0,00)\end{array}$ & $\begin{array}{c}15,1 \\
(0,07)\end{array}$ & $\begin{array}{c}2.428,2 \\
(11,41)\end{array}$ & $\begin{array}{c}4.489,2 \\
(18,83)\end{array}$ & $\begin{array}{c}630,5 \\
(2,49)\end{array}$ & $\begin{array}{c}11.640,6 \\
(54,69)\end{array}$ & - & $\begin{array}{l}2.182,1 \\
(10,25)\end{array}$ \\
\hline XVI. & " & $\ldots \ldots$ & $\begin{array}{r}13,6 \\
(0,07)\end{array}$ & $\begin{array}{c}205,1 \\
(1,09)\end{array}$ & $\begin{array}{c}5.405,6 \\
(28,78)\end{array}$ & $\begin{array}{l}3.640,0 \\
(19,38)\end{array}$ & $\begin{array}{c}3.694,7 \\
(19,67)\end{array}$ & $\begin{array}{l}2,025,9 \\
(10,79)\end{array}$ & $\begin{array}{r}19,9 \\
(0,10)\end{array}$ & $\begin{array}{l}3.778,6 \\
(20,12)\end{array}$ \\
\hline XIV. & $"$ & $\cdots \cdots$ & $\begin{array}{r}0,0 \\
(0,00)\end{array}$ & $\begin{array}{r}2,0 \\
(0,02)\end{array}$ & $\begin{array}{c}104,1 \\
(0,80)\end{array}$ & $\begin{array}{r}74,8 \\
(0,58)\end{array}$ & - & $\begin{array}{l}1,843,5 \\
(14,22)\end{array}$ & $\begin{array}{c}10.934,4 \\
(84,35)\end{array}$ & $\begin{array}{r}4,6 \\
(0,04)\end{array}$ \\
\hline I. & " & $\cdots \cdots$ & $\begin{array}{c}367,8 \\
(3,29)\end{array}$ & $\begin{array}{r}5,6 \\
(0,05)\end{array}$ & $\begin{array}{r}57,0 \\
(0,51)\end{array}$ & $\begin{array}{c}121,2 \\
(1,08)\end{array}$ & $\begin{array}{l}5.917,5 \\
(52,88)\end{array}$ & $\begin{array}{c}197,1 \\
(1,76)\end{array}$ & $\begin{array}{l}3.996,6 \\
(35,72)\end{array}$ & $\begin{array}{c}527,1 \\
(4,71)\end{array}$ \\
\hline XI. & , & $\cdots \cdots$ & $\begin{array}{r}2,0 \\
(0,02)\end{array}$ & $\begin{array}{c}46,0 \\
(0,45)\end{array}$ & $\begin{array}{l}1.128,2 \\
(11,11)\end{array}$ & $\begin{array}{l}3.330,4 \\
(32,80)\end{array}$ & $\begin{array}{c}701,7 \\
(6,91)\end{array}$ & $\begin{array}{c}638,9 \\
(6,29)\end{array}$ & $\begin{array}{l}1.644,8 \\
(16,20)\end{array}$ & $\begin{array}{c}2,659,3 \\
(26,20)\end{array}$ \\
\hline
\end{tabular}




\section{CUADRO VIII}

PRINCIPALES SECCIONES DE EXPORTACION POR PROVINCIA. TRIENIO 1980-82 (Millones de pesetas)

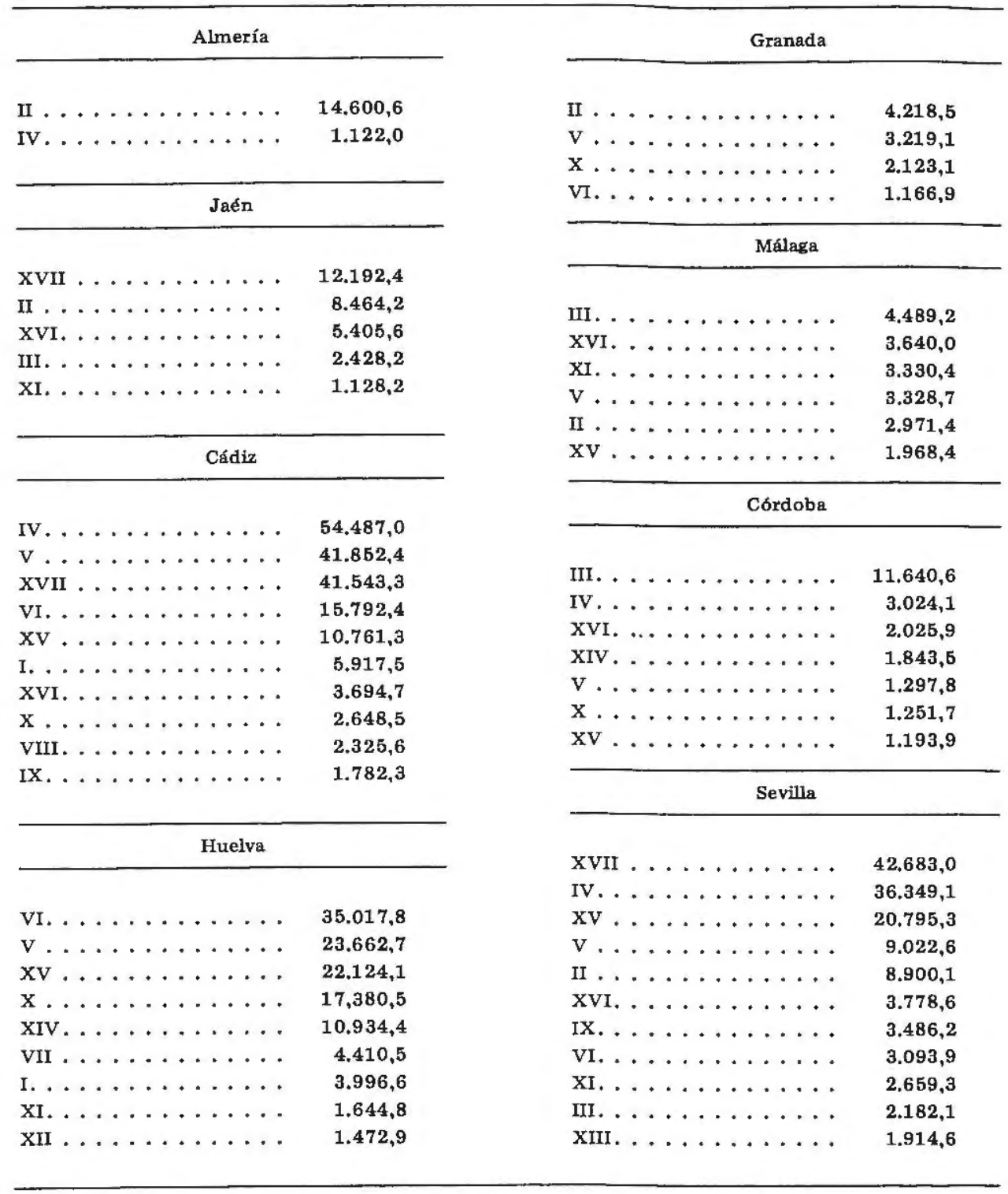


Se refuerza la idea antes expuesta de que en buena medida las exportaciones se originan en unos pocos enclaves. Las exportaciones de primeras materias poco elaboradas y los enclaves industriales, orientados a la exportación, se concentran en unas zonas localmente definidas, sin que tenga lugar una vinculación con otras actividades productivas locales. Hay, pues, un fenómeno de fuerte concentración espacial de las exportaciones dentro de Andalucía, que puede ser explicable en términos de la forma del crecimiento económico de la misma.

En el cuadro VII puede verse, para las principales secciones de las que se exporta, cómo se distribuyen provincialmente.

Aunque algunas secciones, como XVI: "Máquinas y aparatos" se reparte la producción exportadora entre las provincias, recordemos que se trata de secciones no muy importantes en el total de exportaciones. Como vimos, hay una concentración apreciable - casi el 80 por ciento- en las seis primeras secciones, bastando en las restantes que exista una empresa significativa localizada en una provincia, para que aparezca ésta con un elevado porcentaje de participación en el total de la sección, aunque en términos absolutos se trate de una cifra de exportación escasamente relevante, ya que sólo pueden considerarse importantes las seis primeras secciones.

En el cuadro VIII se han recogido las principales secciones de exportación por provincia, que superen los 1.000 millones para el trienio. Se han incluido algunas secciones que no figuraban en el cuadro VII, pero que cumplen con el criterio de superar la cifra adoptada.

Aparte de reflejarse la composición exportadora de cada provincia, según las líneas apuntadas anteriormente, es apreciable un mayor número de secciones que superan los 1.000 millones, en las provincias de Cádiz, Huelva y Sevilla, con lo que estas provincias poseen no sólo los productos que suponen los mayores volúmenes de exportación, sino también la mayor diversificación exportadora.

\section{LAS EXPORTACIONES ANDALUZAS EN EL CONTEXTO ESPAÑOL}

En el cuadro IX efectuamos una comparación de las exportaciones andaluzas y españolas por secciones, para el trienio $80 / 82$.

Las exportaciones andaluzas representan el 10 por ciento de las españolas, porcentaje que es prácticamente el mismo para cada uno de los tres años considerados.

En la última columna del cuadro se recoge la relación (A/E por ciento) entre exportaciones andaluzas y españolas; las secciones en la que la participación andaluza es más importante son las siguientes. La IV: "Productos de 
industrias alimenticias", que incluye las bebidas, con el 30,20 por ciento del total de la sección; esta sección supone, sin embargo, sólo el 5,75 por ciento de las exportaciones españolas. Igual ocurre con la II: "Productos del reino vegetal", que representa el 22,08 por ciento del total de la sección la cual, a su vez, no es importante (1,71 por ciento) en el conjunto de las exportaciones españolas.

Las secciones V y VI: "Productos minerales" y "Productos de las industrias químicas", participan de forma destacada en el total de las exportaciones españolas, con un 8,92 y 6,25 por ciento respectivamente. Andalucía, a su vez, produce el 16,42 y 16,43 por ciento de esas exportaciones.

También participa Andalucía en un 10,92 por ciento en las exportaciones de la sección X: "Materias utilizadas para la fabricación de papel" (3,86 por ciento de las exportaciones españolas).

Por último, quisiéramos destacar dos datos. Uno, la participación de Andalucía en el 12,97 por ciento de las exportaciones españolas de "Material de transporte" (sección XVII, 13,32 por ciento de las exportaciones españolas).

\section{CUADRO IX}

EXPORTACIONES ANDALUCIA Y ESPAÑA POR SECCIONES. TRIENIO 1980-82 (Millones de pesetas)

\begin{tabular}{|c|c|c|c|c|c|}
\hline & Andalucía & $\begin{array}{l}\text { Total } \\
(\% / 0)\end{array}$ & España & $\begin{array}{l}\text { Total } \\
(\% / 0)\end{array}$ & $\begin{array}{c}A / E \\
(0 / 0)\end{array}$ \\
\hline I. . . . . . . . & $11.189,91$ & 2,01 & $90.114,92$ & 1,59 & 12,42 \\
\hline II ............ & $41.116,26$ & 7,40 & $490.436,43$ & 8,69 & 8,38 \\
\hline III. . . . . . . . . & $21.285,71$ & 3,83 & $96.422,63$ & 1,71 & 22,08 \\
\hline IV $\ldots \ldots \ldots \ldots \ldots$ & $98.073,57$ & 17,64 & $324.713,03$ & 5,75 & 30,20 \\
\hline$v \ldots \ldots \ldots \ldots$ & $82.722,51$ & 14,88 & $503.799,88$ & 8,92 & 16,42 \\
\hline vг. ......... & $58.017,58$ & 10,44 & $353.153,10$ & 6,25 & 16,43 \\
\hline vII $\ldots \ldots \ldots \ldots$ & $5.058,08$ & 0,91 & $204.829,51$ & 3,62 & 2,47 \\
\hline vir. . . . . . . . . & $2.577,99$ & 0,46 & $99.979,62$ & 1,77 & 2,58 \\
\hline IX. . . . . . . . & $6.239,87$ & 1,12 & $80.365,00$ & 1,42 & 7,76 \\
\hline$x \ldots \ldots \ldots \ldots$ & $23.845,14$ & 4,29 & $218.323,78$ & 3,86 & 10,92 \\
\hline$x I \ldots \ldots \ldots \ldots$ & $10.151,36$ & 1,83 & $272.130,71$ & 4,82 & 3,73 \\
\hline xII $\ldots \ldots \ldots \ldots$ & $1.898,10$ & 0,34 & $169,427,74$ & 3,00 & 1,12 \\
\hline xıII. . . . . . . . & $2.840,27$ & 0,51 & $148.219,17$ & 2,63 & 1,92 \\
\hline xIv........... & $12.963,40$ & 2,33 & $65.244,68$ & 1,16 & 19,87 \\
\hline$x v \ldots \ldots \ldots \ldots$ & $57.233,81$ & 10,30 & $898,978,16$ & 15,94 & 6,37 \\
\hline xvi. . . . . . . . & $18.783,45$ & 3,38 & 729.572 .78 & 12,93 & 2,57 \\
\hline xvil $\ldots \ldots \ldots \ldots$ & $97.437,01$ & 17,53 & $751.437,11$ & 13,32 & 12,97 \\
\hline xviri........... & $1.870,21$ & 0,34 & $32.029,28$ & 0,57 & 5,84 \\
\hline $\operatorname{xix} \ldots \ldots \ldots \ldots$ & 520,80 & 0,10 & $13.809,46$ & 0,24 & 3,77 \\
\hline$x x \ldots \ldots \ldots$ & $2.015,95$ & 0,36 & $82.380,75$ & 1,46 & 2,45 \\
\hline$x x r \ldots \ldots \ldots \ldots$ & - & 0,00 & $3.892,85$ & 0,07 & 0,00 \\
\hline Total . . . . . . . . . & $555.847,05$ & 100,00 & $5.640 .909,00$ & 100,00 & 9,85 \\
\hline
\end{tabular}


$\mathrm{Y}$, por otra parte, la reducida participación en las secciones XV y XVI: "Metales comunes y sus manufacturas" y "Máquinas y aparatos, material eléctrico", es especialmente relevante la reducida presencia en esta última sección (sólo un 2,57 por ciento), que representa el 13 por ciento de las exportaciones españolas.

En suma, la composición de las exportaciones andaluzas no difiere, en general, sustancialmente del tipo de exportaciones del conjunto de la economía española.

Este hecho puede ser apreciado con más precisión en el cuadro X, donde se comparan las exportaciones andaluzas con las del resto del Estado español. La tónica general es de una cierta similitud en la orientación exportadora, sin embargo, hay diferencias notables que conviene destacar.

\section{CUADRO X}

EXPORTACIONES ANDALUCIA Y RESTO ESPAÑA POR SECCIONES. TRIENIO 1980-82 (Millones de pesetas)

\begin{tabular}{|c|c|c|c|c|}
\hline & Andalucía & $\begin{array}{l}\text { Total } \\
(\% / 0)\end{array}$ & Resto España & $\begin{array}{l}\text { Total } \\
(\% / 0)\end{array}$ \\
\hline I. $\ldots \ldots \ldots \ldots$ & $11.189,91$ & 2,01 & $78.925,01$ & 1,55 \\
\hline II $\ldots \ldots \ldots \ldots$ & $41.116,26$ & 7,40 & $449.320,17$ & 8,84 \\
\hline III. . . . . . . . . & $21.285,71$ & 3,83 & $75.136,92$ & 1,48 \\
\hline IV $\ldots \ldots \ldots \ldots$ & $98.073,57$ & 17,64 & $226,639,98$ & 4,46 \\
\hline$v \ldots \ldots \ldots$ & $82.722,51$ & 14,88 & $421.077,37$ & 8,28 \\
\hline vл ........... & $58.017,18$ & 10,44 & $295.135,52$ & 5,80 \\
\hline VII $\ldots \ldots \ldots \ldots$ & $5.058,08$ & 0,91 & $199.771,43$ & 3,93 \\
\hline virr. . . . . . . . . & $2.577,99$ & 0,46 & $97.401,63$ & 1,92 \\
\hline IX $\ldots \ldots \ldots \ldots$ & $6.239,87$ & 1,12 & $74.125,13$ & 1,46 \\
\hline$x \ldots \ldots \ldots$ & $23.845,14$ & 4,19 & $194.478,64$ & 3,82 \\
\hline$x r \ldots \ldots \ldots \ldots$ & $10.151,36$ & 1,83 & $261.979,35$ & 5,15 \\
\hline $\mathrm{XII} \ldots \ldots \ldots \ldots$ & $1.898,10$ & 0,34 & $167.529,64$ & 3,29 \\
\hline XIII. . . . . . . . . & $2.840,27$ & 0,51 & $145.378,90$ & 2,86 \\
\hline xIv ............. & $12.963,40$ & 2,33 & $52.261,28$ & 1,03 \\
\hline$x v \ldots \ldots \ldots$ & $57.233,82$ & 10,30 & $841.744,34$ & 16,56 \\
\hline xvi. . . . . . . . & $18.783,45$ & 3,38 & $710.789,93$ & 13,98 \\
\hline xVII $\ldots \ldots \ldots \ldots$ & $97.437,01$ & 17,53 & $654.000,10$ & 12,86 \\
\hline 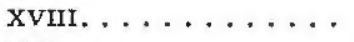 & $1.870,21$ & 0,34 & $30.159,07$ & 0,59 \\
\hline $\operatorname{xrx} \ldots \ldots \ldots$ & $\mathbf{5 2 0 , 8 0}$ & 0,10 & $13.288,66$ & 0,26 \\
\hline $\mathrm{xx} \ldots \ldots \ldots$ & $2.015,95$ & 0,36 & $80.364,80$ & 1,58 \\
\hline xxi. . . . . . . & - & 0,00 & $3.892,85$ & 0,07 \\
\hline Total ........... & $555.847,05$ & 100,00 & $5.085 .061,95$ & - \\
\hline
\end{tabular}


Andalucía tiene un mayor peso exportador en las secciones IV: "Productos de las industrias alimenticias" (que incluye bebidas) (17,64 por ciento); V: "Productos minerales" (14,88 por ciento); VI: "Productos de las industrias químicas y conexas" (10,44 por ciento); y XVII: "Material de transporte" (17,53 por ciento), esto es, como hemos venido constatando, exportaciones de primeras materias de escasa elaboración y algunos enclaves industriales. Las exportaciones españolas comparten aproximadamente estas características; sin embargo, hay algunas diferencias notables. La sección VII: "Materias plásticas artificiales", tiene una importancia del 3,93 por ciento en las exportaciones españolas, mientras que en las andaluzas no llega al 1 por ciento. Igual ocurre con la sección XV: "Metales comunes y sus manufacturas", es importante en Andalucía (10,30 por ciento de las exportaciones), aunque no tanto como en el resto de España (16,55 por ciento). Por último, la sección XVI: "Máquinas y aparatos, material eléctrico", es casi un 14 por ciento de las exportaciones del resto de España y sólo el 3,38 de las andaluzas.

Estos datos ponen de manifiesto la carencia en Andalucía de una industria productora y exportadora de manufacturas de industria ligera, que sí ha tenido un cierto desarrollo en el resto de España; en este sentido, el carácter dual de la economía andaluza destaca sobre el del resto de la economía española. Tal vez esta sea la principal conclusión de la comparación que hemos efectuado. 